\title{
A196 ANTI-MIT3 ANTIBODIES IN SYSTEMIC SCLEROSIS
}

Cavazzana I, ${ }^{1}$ Taraborelli $\mathrm{M}_{1}{ }^{1}$ Ceribelli $\mathrm{A}_{1}{ }^{2}$ Fredi $\mathrm{M}_{1}{ }^{1}$ Norman $\mathrm{G},{ }^{3}$ Satoh $\mathrm{M}_{1}{ }^{4}$ Franceschini $\mathrm{F}^{1}{ }^{1}$ Rheumatology Unit and Chair, Spedali Civili, University of Brescia, Italy; ${ }^{2}$ Department of Oral Biology, University of Florida, Gainesville, Florida, USA; ${ }^{3}$ INOVA Diagnostics, San Diego, California, USA; ${ }^{4}$ Department of Medicine, University of Florida, Gainesville, Florida, USA

\subsection{6/ard.2010.149021.6}

Background and objectives Antimitochondrial (AMA) is considered the serological hallmark of primary biliary cirrhosis (PBC). Other autoantibodies recognising nuclear dots (Sp100) and nuclear pore complex proteins (gp-210) are associated to severe $\mathrm{PBC}$, but they are found by less available methods. An ELISA with a combination of three mitochondrial antigens (MIT3), Sp100 and gp-210 has been recently developed. The aim of our study was to analyse the prevalence, associations and the fine specificity of antibodies to MIT3, Sp100 and gp210 in a cohort of Italian patients affected by systemic sclerosis (SSc).

Materials and methods 201 sera were analysed by ELISA (Quanta Lite TM ELISA; INOVA Diagnostics Inc, San Diego, California, USA) for the detection of antibodies to MIT3 (using goat anti-human IgA and IgG antibodies), Sp100 and gp210. Antinuclear, anti-ENA and anti-RNA polymerase III were detected by indirect immunofluorescence (IIF), counterimmunoelectrophoresis and ELISA, respectively. AMA were identified by IIF on rodent kidney/stomach/liver tissue sections. The diagnosis of SSc and $\mathrm{PBC}$ were assessed according to LeRoy criteria and EASL Clinical Practice Guidelines. More than $99 \%$ of patients were Caucasians of Italian ancestry.

Results Antibodies to combination of MIT3, gp210 or Sp100 (anti-PBC screen+) were detected in $21 \%$ of cases (43 sera): anti-MIT3 were found in 36, anti-Sp100 in 5 and anti-gp210 in 1 serum. Anticentromere (ACA) and AMA were more frequently detected in anti-PBC screen + when compared with 158 negative group $(p=0.0005$ and $p=0.001)$. When the authors considered only $\mathrm{ACA}+$ patients, $\mathrm{AMA}$ and $\mathrm{PBC}$ were more frequently found in anti-PBC screen + cases $(p=0.02$ and $p=0.0009$ ). Analysing the anti-MIT3 isotypes (36 sera), the authors found isolated $\operatorname{IgG}$ in $44.5 \%$, IgA in $33.4 \%, \operatorname{IgG}+\operatorname{Ig} A$ in $22 \%$. Autoantibodies and clinical features of SSc didn't show a different distribution between groups, except for skin ulcers and pulmonary hypertension more frequently detected in isolated IgG and in total IgG anti-MIT3 cases, respectively. AMA were more frequently detected in $\operatorname{Ig} A+\operatorname{Ig} G$ versus $\operatorname{Ig} A$ or IgG anti-MIT3 groups $(p=0.005$ and $p=0.002)$. Ig $A+\operatorname{IgG}$ antiMIT3 showed a more frequent diagnosis of $\mathrm{PBC}$ and elevation of serum ALP (considered a marker of liver disease severity) despite of urso-deoxycholic acid treatment, when compared with others $(p=0.014$ and $p=0.04)$. Anti-MIT3 antibodies showed a good sensitivity and specificity $(75 \%$ and $85 \%$, respectively) for $\mathrm{PBC}$ diagnosis.

Conclusions The availability of fully automated ELISA could enhance the possibility of finding different autoantibodies considered markers of $\mathrm{PBC}$ in routine laboratory analysis, avoiding assays with diversified antigen sources. The anti-MIT3 isotypes characterisation could improve the assessment of patients with $\mathrm{PBC}$, with higher risk of disease severity. 